\title{
Brolucizumab—another anti-VEGF or beyond
}

\author{
Ashish Sharma ${ }^{1} \cdot$ Nikulaa Parachuri $^{1} \cdot$ Nilesh Kumar $^{1}{ }^{1} \cdot$ Rohini Sharma $^{2} \cdot$ Francesco Bandello $^{3}$. \\ Baruch D. Kuppermann ${ }^{4}$ - Anat Loewenstein ${ }^{5}$
}

Received: 26 March 2020 / Revised: 8 April 2020 / Accepted: 8 April 2020 / Published online: 21 April 2020

(c) The Royal College of Ophthalmologists 2020

There are quite a few anti-VEGFs that are used in ophthalmic practice to treat a variety of conditions. Ranibizumab \{(Lucentis; Genentech, South San Francisco, CA) (Razumab; Intas Pharmaceutical Ltd, Ahmedabad, India * Biosimilar of ranibizumab approved only in India) Bevacizumab (Avastin; Genentech, South San Francisco, CA) and Aflibercept (Eylea; Regeneron, Tarrytown, NY)\} are currently being used in the management of diabetic macular oedema (DME), uveitic macular oedema, retinal vein occlusions and neovascular age related macular degeneration (nAMD) [1]. Is the recently FDA approved brolucizumab (Beovu, Novartis) just another drug in the armamentarium of anti-VEGFs (vascular endothelial growth factor) or does it have something else to offer [2]?

Pegaptanib sodium, the first anti-VEGF approved, is a pegylated ribonucleic acid aptamer which binds near the heparin-binding domain of VEGF-A, thus preventing VEGF $_{165}$, making it a selective anti-VEGF agent. Ranibizumab, the second anti-VEGF agent approved for the treatment of nAMD is a chimeric molecule derived from a murine full-length monoclonal antibody which binds to, and inhibits, the biologic activity of all active forms of VEGF-A. Razumab is the biosimilar of ranibizumab,

$\triangle$ Ashish Sharma

drashish79@hotmail.com

1 Lotus Eye Hospital and Institute, Avinashi Road, Coimbatore, TN, India

2 Department of Healthcare, The TIPS Global Institute, Coimbatore, TN, India

3 University Vita-Salute, Scientific Institute San Raffaele, Milano, Italy

4 Gavin Herbert Eye Institute, University of California, Irvine, CA, USA

5 Division of Ophthalmology, Tel Aviv Sourasky Medical Center and Sackler Faculty of Medicine, Tel Aviv University, Tel Aviv, Israel which is a recombinant humanized $\operatorname{IgG1}$ monoclonal antibody fragment. Bevacizumab, an anticancer drug with a larger size and lower affinity also inhibits all isoforms of VEGF and is being used in a small dose. Aflibercept is a VEGF Trap-eye that binds to all VEGF-A and VEGF-B isoforms as well as to placental growth factor. The latest drug brolucizumab is a humanized single-chain antibody fragment inhibitor of all isoforms of vascular endothelial growth factor-A (VEGF-A) and has a molecular weight of just $26 \mathrm{kDa}$ when compared to its predecessors, bevacizumab $(149 \mathrm{kDa})$, ranibizumab $(48 \mathrm{kDa})$ and aflibercept $(115 \mathrm{kDa})$. This is the smallest monoclonal antibody ever made in medicine [3].

With the current focus being on reducing the injection burden for patients, the number of anti-VEGF injections needed in a year to maintain vision becomes the primary point of interest. The recommended monthly dosing schedule and the treat and extent regimen are currently being followed for ranibizumab. Three monthly loading doses followed by injections at a fixed interval of 8 weeks is the recommended dosing schedule for aflibercept. Bevacizumab is being used off label either as monthly injections or by the pro-re-nata protocol [4]. For brolicizumab, the FDA has approved a 12-week dosing schedule after 3 monthly loading doses. So, it becomes the only anti-VEGF drug that goes beyond the 8 -week interval and has the least number of injections in a year leading to a reduced financial burden on the patients according to the results of the HAWK and HARRIER trial [5].

Brolucizumab gives a tighter fluid control as evident from the central subfield thickness graph obtained from the results of the HAWK and HARRIER trials. Smaller size and higher molecular concentration might help to increase the duration of fluid control. The pre-clinical data revealed that the retina had a 2.2 times higher exposure to the molecule when compared to ranibizumab. Retinal pigment epithelium (RPE)/Choroid complex also had 1.7 times higher exposure [5]. None of the prospective trials in the past have differentiated the type of fluid. HAWK and HARRIER have 
analyzed the impact of brolucizumab on intra retinal fluid (IRF)/sub retinal fluid (SRF) and sub-RPE fluid. However, IRF and SRF were not analyzed in isolation. The better control of IRF/SRF/sub-RPE fluid with brolucizumab might have contributed to $>50 \%$ proportion of patients maintaining a q12w dosing through 1 year of follow-up [5]. Brolucizumab goes beyond in fluid control compared to the anti-VEGF's used in the past.

Ocular inflammation has been reported with all the monoclonal anti-VEGF antibodies in use for intra vitreal therapy. Souied et al. have reported an increased number of ocular inflammatory events in patients who received aflibercept when compared to ranibizumab which were mostly mild reactions [6]. Acute intraocular inflammation also known as sterile endophthalmitis was reported more in the bevacizumab group when compared with the ranibizumab group according to the CATT trial. The reasons for which are debated to be due to an immunologic response to the larger and more immunogenic bevacizumab molecule, differences in glycosylation between the two agents, or contaminants (silicone oil) in the solution. However, there is a difference in the inflammation profile of brolucizumab when compared with the inflammation of the other anti-VEGF's as per the recent alert by the American Society of Retina Specialists. There have been 14 cases of vasculitis. Of which, 11 were designated as occlusive retinal vasculitis [7]. It is yet to be seen whether the inflammation pattern is different with brolucizumab compared with other anti-VEGFs in long term with larger data. Prescribing information leaflet for brolucizumab in the US mentions a $4 \%$ rate of intraocular inflammation and a $1 \%$ rate of retinal artery occlusion. The manufacturer has reviewed and stated that the incidence of adverse events remains consistent with or below the package insert [8].

Brolucizumab is a newer class of anti-VEGF drugs and it is beyond the properties of anti-VEGFs being used till date in terms of the structure, function and adverse effects. However, we do not have robust real world data till now. Once we have the data from real world studies, we would be able to say with conviction whether brolucizumab is just another anti-VEGF or beyond.

\section{Compliance with ethical standards}

Conflict of interest AS: Consultant: for Novartis, Allergan, Bayer and Intas. FB: Consultant: Allergan, Bayer, Boehringer- Ingelheim, Fidia Sooft, Hofmann La Roche, Novartis, NTC Pharma, Sifi, Thrombogenics, Zeiss. BDK: Clinical Research: Alcon, Alimera, Allegro, Allergan, Apellis, Clearside, Genentech, GSK, Ionis, jCyte, Novartis, Regeneron, ThromboGenics; Consultant: Alimera, Allegro, Allergan, Cell Care, Dose, Eyedaptic, Galimedix, Genentech, Glaukos, Interface Biologics, jCyte, Novartis, Ophthotech, Regeneron, Revana, Theravance Biopharma. AL reports other from Allergan, other from Novartis, other from Roche, other from Notal Vision, other from Forsights labs, other from Beyeonics, other from Bayer Health Care. NK, NP and RS: None

Publisher's note Springer Nature remains neutral with regard to jurisdictional claims in published maps and institutional affiliations.

\section{References}

1. Tah V, Orlans HO, Hyer J, Casswell E, Din N, Sri Shanmuganathan $\mathrm{V}$, et al. Anti-VEGF therapy and the retina: an update. J Ophthalmol. 2015;2015:627674. https://doi.org/10.1155/2015/627674.

2. Novartis announces FDA filing acceptance and Priority Review of brolucizumab (RTH258) for patients with wet AMD. Novartis. 2020. https://www.novartis.com/news/media-releases/novartis-announcesfda-filing-acceptance-and-priority-review-brolucizumab-rth258-pa tients-wet-am.

3. Sharma A, Kumar N, Kuppermann BD, Bandello F. Brolucizimableading an era of structural revolution for long-term VEGF suppression. Eye. 2020;34:611-3.

4. Lanzetta P, Loewenstein A, and The Vision Academy Steering Committee. Fundamental principles of an anti-VEGF treatment regimen: optimal application of intravitreal anti-vascular endothelial growth factor therapy of macular diseases. Graefes Arch Clin Exp Ophthalmol. 2017;255:1259-73.

5. Dugel PU, Koh A, Ogura Y, Jaffe GJ, Schmidt-Erfurth U, Brown DM, et al. HAWK and HARRIER: Phase 3, multicenter, randomized, double-masked trials of brolucizumab for neovascular agerelated macular degeneration. Ophthalmology. 2020;127:72-84.

6. Souied EH, Dugel PU, Ferreira A, Hashmonay R, Lu J, Kelly SP. Severe ocular inflammation following ranibizumab or aflibercept injections for age-related macular degeneration: a retrospective claims database analysis. Ophthalmic Epidemiol. 2016;23:71-9.

7. Clinical Updates: Beovu Update for ASRS Members. 2020. https://www.asrs.org/clinical/clinical-updates/960/Beovu-Updatefor-ASRS-Members.

8. Novartis Responds to ASRS Note Raising Safety Concerns With Wet AMD Drug Beovu. 2020. https://eyewire.news/articles/nova rtis-responds-to-asrs-note-raising-safety-concerns-of-beovu/. 